\title{
L'analyse du discours sur une problématique environnementale : un projet d'enseignement pour des étudiants en sciences
}

Michel Goldberg et Grâce Kraska

\author{
(2) OpenEdition \\ Journals \\ Édition électronique \\ URL : http://journals.openedition.org/ere/1992 \\ DOI : $10.4000 /$ ere. 1992 \\ ISSN : 2561-2271 \\ Éditeur \\ Centr'ERE
}

Référence électronique

Michel Goldberg et Grâce Kraska, «L'analyse du discours sur une problématique environnementale : un projet d'enseignement pour des étudiants en sciences ", Éducation relative à l'environnement [En ligne], Volume 8 | 2009, mis en ligne le 20 décembre 2009, consulté le 21 février 2020. URL : http:// journals.openedition.org/ere/1992 ; DOI : 10.4000/ere.1992 


\title{
L'analyse du discours sur une problématique environnementale : un projet d'enseignement pour des étudiants en sciences
}

\author{
Michel Goldberg et Grâce Kraska
}

1 L'article que nous proposons relate une expérience de formation destinée aux étudiants de la licence de biochimie de l'Université de La Rochelle. Ce projet vise à donner aux étudiants scientifiques des outils pour participer à des débats de société portant sur des thèmes technologiques. Notre expérience porte sur l'analyse critique de documents traitant de problématiques scientifiques, sociétales et environnementales.

\section{Présentation du projet pédagogique}

L'éducation relative à l'environnement (ERE) est pluridisciplinaire en ce qu'elle porte sur des problématiques qui touchent à la fois aux sciences de la nature et aux sciences sociales, ainsi qu'à des enjeux politiques, éthiques et épistémologiques. Dans notre projet, les étudiants en biochimie, familiers de l'étude de problèmes disciplinaires en sciences de la vie, ont été confrontés à l'étude de textes dans lesquels les savoirs biochimiques étaient intimement insérés dans des problématiques environnementales : elles concernaient la mise en culture de plantes génétiquement modifiées (PGM). Cette technologie a de nombreuses implications environnementales, sociales, économiques, scientifiques et politiques (à titre d'exemple, avec des points de vue très divergents, citons Houdebine, 2000 ; Seralini, 2004 ; Kuntz, 2006 ; Robin, 2008). Les étudiants ont fait face à une difficulté classique des scientifiques impliqués dans des recherches technologiques: la nécessité de comprendre certains enjeux non disciplinaires des projets technologiques. Dans leur formation universitaire en licence de biochimie, cet enseignement était le seul dédié à cette ouverture sur le débat, la pluridisciplinarité et le débat de société. 
3 Nous avons privilégié l'approche d'une problématique environnementale par l'analyse du discours, car tous les savoirs, tous les débats, tous les jugements passent par le discours. Les partenaires de projets environnementaux, issus de disciplines, de cultures, de conceptions philosophiques différentes, n'ont pas d'autre outil plus efficace que la parole pour échanger, et bâtir des projets basés sur des compromis bien compris. Or, les étudiants en sciences, confrontés en permanence à des discours, sont généralement peu ou mal outillés pour repérer les thèses en présence, les courants de pensée, la construction du texte, ses présupposés, ses préjugés, ses sous-entendus, son idéologie, sa rhétorique, sa construction logique, sa subjectivité.

4 L'expérience que nous relatons ici repose sur une démarche pédagogique qui stimule les étudiants pour qu'ils construisent leurs savoirs eux-mêmes, dans une démarche collaborative. Elle s'inscrit dans la suite d'autres études d'analyse de documents menées avec des étudiants en biochimie (Goldberg, 2005 ; Goldberg et al., 2007, 2008).

\section{L'ingénierie pédagogique de notre projet}

5 Les étudiants ont tout d'abord participé à un cours de 3 heures où ont été introduits et discutés quelques concepts pour l'étude critique de textes: les notions d'argumentation, de discussion, d'éthique de la discussion, d'analyse et de jugement ont été discutées. Quelques ouvrages de référence ont été utilisés pour construire cette partie du cours (nous citerons Perelman, 1977 ; Bardin, 1993 ; Danblon, 2005 ; Amossy, 2006 ; Moirand, 2007). Ces ouvrages sont des manuels classiques pour l'étude critique du contenu des textes (analyse de contenu, analyse du discours, rhétorique, discours et démocratie).

6 Avec l'enseignant, les étudiants ont construit deux grilles de lecture selon 13 critères : (1) une grille d'analyse de contenu et (2) une grille dans laquelle les étudiants sont conduits à porter un jugement sur l'argumentation du document. Ces critères portaient principalement sur le thème, la thèse, le plan, l'énonciation, l'argumentaire du texte. Les étudiants ont ensuite disposé de cinq jours pour la mise en ligne de leurs analyses sur la plate-forme électronique Moodle.

7 Lors de la leçon suivante, les étudiants ont pris connaissance d'un texte qui traite du débat de société sur les organismes génétiquement modifiés. Il est extrait d'un ouvrage de Jean-Paul Oury (2006), La querelle des OGM. Les étudiants ont analysé ce texte en classe collectivement, par petits groupes de trois. Le texte a ensuite été discuté en séance plénière lors du cours suivant. Des concepts et des outils pour l'analyse de textes ont été rediscutés et précisés (l'implicite, le sous-entendu, le sens littéral et le sens connoté des termes, l'ironie, l'analogie). Enfin, les petits groupes de trois étudiants ont rédigé un projet de lettre destinée à l'auteur du document. Ils ont choisi un rôle fictif d'énonciateur (soit celui de l'éditeur de l'ouvrage qui émet un avis sur le manuscrit, soit celui du représentant d'une association de consommateurs rétive à la mise en culture des PGM). Les étudiants ont décidé de l'objectif visé par cette lettre et ont rédigé ensuite quatre ou cinq paragraphes qui concernaient l'argumentation, la valeur des informations, le style et l'éthique de l'argumentation du texte de Jean-Paul Oury. Dans cette lettre, les étudiants étaient invités à prendre position sur la conception qu'ils avaient de l'éthique de l'argumentation (par exemple sur la logique de l'argumentation, les références extra-rhétoriques, la prise en compte des positions des autres partenaires du débat, le refus des attaques ad hominem). 


\section{Le texte analysé par les étudiants était une fiction : une France qui aurait banni les OGM}

8 Nous avons choisi un texte répondant à plusieurs critères. Il s'agit d'un texte (1) concernant une problématique actuelle, (2) traitant d'un problème lié aux technologies intéressant le biochimiste, (3) ayant des implications sociales, économiques et environnementales, (4) paru chez un éditeur réputé (les Presses Universitaires de France). Il doit porter sur (5) un problème relativement connu des étudiants, et il doit s'agir (6) d'un texte polémique afin d'éveiller l'intérêt de nos étudiants. Nous avons choisi un texte (de 11 paragraphes) intitulé "L'exception culturale », ou comment mettre un terme à la querelle [des OGM] de manière radicale (Oury, 2006, p. 18-22). Il s'agit, selon l'auteur, d'une expérience de pensée qui vise à décrire la situation d'un "pays [qui] décide d'interdire la technologie [OGM] sur son territoire». Pour permettre au lecteur de suivre notre présentation de ce projet, nous avons choisi de présenter ici un paragraphe qui rend compte de la tonalité de l'ensemble. Voici l'extrait en question.

Pour ce qui concerne maintenant les campagnes, on peut penser que les agriculteurs, informés des performances techniques et des succès remportés par leurs homologues dans les pays voisins, seraient tentés d'importer et de planter des semences GM en fraude ${ }^{1}$. La milice serait chargée d'intercepter cette contrebande, en veillant aux frontières, mais également de détecter les fermes qui ont contrevenu à la règle en plantant les semences interdites. Ce travail difficile ne s'effectuerait pas sans bavures. En effet, on peut imaginer que la tâche qui consiste à détecter les champs de PGM illégaux, donnerait lieu à de nombreuses méprises. Afin d'avoir plus d'indices, on peut supposer que la milice en question n'hésiterait pas à recourir à des dénonciations. Le champ suspect, quant à lui, serait détruit sans plus d'examen, et l'agriculteur encourrait une peine de prison exemplaire. Quant à sa famille, elle subirait la honte d'être montrée du doigt dans tout le village. (Oury, 2006, p. 18-22)

\section{La discussion du texte de J.-P. Oury avec les étudiants}

\section{Le grand respect des étudiants pour les textes écrits par des universitaires}

Nous traiterons ici d'un problème qui nous semble intimement lié à l'éducation relative à l'environnement en ce qu'il met en évidence une grande difficulté des étudiants pour émettre un jugement critique dans un débat portant sur une problématique environnementale, pour peu qu'elle concerne un ouvrage paru chez un éditeur universitaire.

10 Nous ne porterons pas ici de jugement sur la valeur des travaux critiques réalisés par les étudiants. Nous relèverons certaines difficultés qu'ils ont pu rencontrer dans leur analyse de discours ainsi que certaines aptitudes qu'ils ont montrées pour mener à bien ce travail critique. Ces aptitudes n'apparaissent parfois qu'au terme de patientes discussions, car de nombreux étudiants se montrent rétifs à l'analyse critique de la fiction écrite par Jean-Paul Oury.

11 Les étudiants témoignent d'un grand respect pour les textes qui leur sont remis dans le contexte de leur formation scientifique, un respect qui confine parfois au refus de porter un regard critique. Généralement, dans les premiers cycles universitaires, ces 
documents sont des démonstrations, des descriptions de méthodes d'analyse ou encore des articles scientifiques majeurs. Classiquement, ces textes doivent être appris, compris, connus. Ils constituent des modèles de pensée pour les étudiants. Or, on oublie trop souvent que la pensée critique peut aussi s'exercer sur ces textes-là, comme nous l'avons montré dans nos publications référencées plus haut. Il n'est pas étonnant que de nombreux étudiants aient été quelque peu déroutés par la consigne qui consistait à mener une lecture critique du texte de J.-P. Oury. Ils ont rencontré des difficultés pour se mettre en posture de faire l'analyse du discours d'un texte abordant des problèmes sociaux et scientifiques. Ce travail devait passer par une phase d'annotation du document en vue de l'analyser. Certes, l'étudiant a l'habitude de surligner les termes importants ou difficiles, mais il n'exprime pas aisément ses étonnements, ses doutes, ses oppositions. En conséquence, l'enseignant a dû insister et donner l'exemple pour que les étudiants annotent le texte de mentions qui témoignent d'une première forme d'analyse qui facilite la construction d'un jugement synthétique. Peu à peu, les étudiants se sont autorisés à écrire dans la marge du texte de petites notes telles que «style ! », « lien logique ? », « abus de langage ? ", « évidence », « info+++ ». Il apparaît que le questionnement d'un texte, le relevé de ses incohérences, de ses aspects stylistiques, de son argumentaire, de son énonciation constituent des démarches inhabituelles dans la formation universitaire de l'étudiant scientifique. L'analyse de discours est une démarche critique difficile, car nouvelle dans ce contexte. Elle exige l'utilisation d'une méthodologie qui s'appuie sur un travail d'étude et d'apprentissage que les étudiants ont connu au collège et au lycée, et dont ils doivent souvent réapprendre ou réactiver les mécanismes.

\section{Des concepts inconnus, méconnus ou oubliés pour procéder à une analyse de texte}

L'étude critique en classe révèle que de nombreux étudiants situent avec précision certains aspects du texte qui suscitent un malaise, mais ils éprouvent de grandes difficultés pour l'exprimer clairement. Ils ignorent ou connaissent mal certains des concepts qui permettent d'exprimer clairement une pensée. Dans l'étude du texte qui nous occupe, des étudiants ont ainsi eu beaucoup de peine pour exprimer la présence d' analogies abusives (ou amalgames). Ce repérage implique un jugement de valeur sur l'argumentation de l'auteur, et cette démarche est inhabituelle et difficile à réaliser dans le cadre d'une formation scientifique centrée sur les connaissances disciplinaires. La différence entre des sous-entendus et des présupposés n'était pas claire non plus pour de nombreux étudiants. Nous avons aussi été étonnés d'observer une grande confusion entre le thème et la thèse, deux notions qui sont pourtant classiquement enseignées au lycée, et dont la pratique gagnerait à être réactivée dans l'enseignement supérieur pour les étudiants scientifiques.

\section{Un sentiment d'infériorité chez les étudiants qui limite leur aptitude à critiquer un texte de science}

13 En classe, dans plusieurs groupes, des étudiants expriment la difficulté de juger de la valeur informative d'un texte qui ne contient... aucune information factuelle, statistique, ni aucune référence bibliographique. Plusieurs étudiants expriment que l'absence flagrante d'informations sérieuses constitue un problème, mais ils le 
ressentent comme une faiblesse $d u$ lecteur (comme si ces informations manquantes étaient tellement évidentes qu'elles n'auraient pas à être formulées par l'auteur de l'ouvrage). Le respect de l'auteur, de la maison d'édition, du préfacier, empêche de repérer la faiblesse flagrante de l'argumentaire de l'auteur, qui repose pour l'essentiel sur son imaginaire.

14 Pour certains étudiants, il est proprement inimaginable qu'un tel texte, paru chez un éditeur reconnu, et soumis à un comité de lecture, puisse ne contenir aucune information extra-rhétorique solide. Ils sont donc conduits à trouver ces informations à tout prix, et se contentent donc de maigres données rapportées par l'auteur (et qui se situent à la marge de la problématique de la mise en culture des OGM).

\section{Une difficulté des étudiants pour exprimer leurs doutes}

\section{texte explicatif. C'est un texte d'explication. L'auteur ne prend jamais position, il reste objectif.} Il expose les risques, les conséquences, ou les faits probables pouvant arriver suite à une idée. Il illustre son explication par des arguments de poids facilement imaginables et tout à fait plausibles. explication alors qu'il s'agit d'un texte polémique qui combat une position politique extrême, quasiment inaudible en France, selon laquelle il faudrait interdire toute recherche, tout développement technologique et toute diffusion d'information scientifique sur les OGM. Les étudiants expriment la difficulté de classer ce texte dans une catégorie (discussion ou explication), et pourtant... ils se résignent tous à le faire, sans repérer l'ambiguïté du texte. Ils obéissent en fait à la consigne qui consistait à classer cet article. Fallait-il le classer coûte que coûte ? Et dans ce cas, ne pouvait-on exprimer les raisons de ce classement et également les doutes et les difficultés rencontrées? Il est intéressant de noter qu'un certain nombre d'étudiants ne se sont pas autorisés à exprimer un doute qui était pourtant présent à leur esprit.

\section{Une confrontation avec les textes dans les discussions en petits groupes}

Malgré les difficultés mentionnées ci-dessus pour l'analyse du texte de J.-P. Oury, de nombreuses remarques orales des étudiants témoignent de leur volonté de débattre de l'argumentaire de l'auteur. Certains étudiants notent que l'auteur aurait pu, en utilisant sa seule imagination (comme il le fait dans le texte analysé), proposer une 
vision idyllique d'un État sans OGM ou au contraire une vision mortifère d'un État qui contraindrait l'ensemble des agriculteurs à l'usage des OGM. La valeur de l'expérience de pensée de l'auteur est ainsi remise en question comme outil pour prévoir les dangers d'une politique.

Dans ce même texte, de nombreuses allusions à une France occupée par un pouvoir totalitaire, dictatorial et obscurantiste, ainsi qu'à une sorte de Résistance suscitent aussi un débat parmi les étudiants. Le plus souvent, ils rejettent ces amalgames, considérant qu'ils discréditent en fait par leur excès l'auteur qui les énonce. Cependant, quelques étudiants voient dans ce procédé rhétorique une voie intéressante pour éveiller la conscience du lecteur.

L'énonciation est aussi discutée par certains groupes d'étudiants. Ils écrivent par exemple que l'auteur «ne s'exprime jamais en son propre nom ». Ils remarquent que l'auteur présente sa fiction comme une description «objective ", comme s'il s'agissait d'une sorte de démonstration, sans marque de l'identité et de la position d'un énonciateur.

\section{Discussion}

La formation critique, l'aptitude à construire un argumentaire, à débattre et à émettre un jugement sont pleinement constitutifs d'une éducation relative à l'environnement pour les étudiants en biochimie, car cette discipline est partie prenante de nombreux projets liés à l'environnement (dans l'industrie agro-alimentaire, en médecine, en cosmétologie, voire dans l'industrie de l'armement). Cette expérience témoigne des difficultés que rencontrent les étudiants en licence de sciences de la vie pour intégrer ces pratiques. Notre projet a mis en lumière la nécessité de développer dans la formation universitaire la lecture critique et le débat sur les technologies ayant un impact environnemental, sociétal ou politique.

Les étudiants se sont vu proposer des outils d'analyse du discours et ont pris connaissance de certains questionnements éthiques qui contribuent à la formation de leur pensée critique. Nous avons montré ci-dessus que l'acquisition de certains concepts utiles pour développer la critique du discours, ainsi que la mise en place d'un contexte propice au développement de la pensée critique permettent aux étudiants d'analyser et de porter un jugement argumenté sur un texte polémique traitant de technologie et d'impact social, écologique et politique. Cette expérience est poursuivie à l'Université dans le contexte même du cours d'enzymologie, tant dans la partie théorique que dans l'étude des applications industrielles.

Dans ce projet, notre démarche pédagogique a pris en compte une dimension ternaire du temps : (1) le cours en face à face est construit sur des mini activités qui stimulent la collaboration et la production; (2) l'après-cours offre le temps de la réflexion en différé avec un questionnement envoyé à chaque étudiant par le biais de la plate-forme électronique et (3) ce temps de maturation et de production prépare le cours suivant et devient ainsi une amorce stimulante. En s'extrayant de la réactivité immédiate induite souvent lors d'un cours, ce temps de maturation et de production prépare le cours suivant et devient ainsi une amorce stimulante.

Cette formation n'a pratiquement pas d'équivalent dans la formation scientifique universitaire classique, et nous ne pouvons que le regretter, car les biochimistes sont 
confrontés dans leurs recherches à des problèmes sociaux, environnementaux, politiques, éthiques auxquels il est difficile de rester indifférent, et face auxquels il importe de disposer d'outils d'analyse.

\section{BIBLIOGRAPHIE}

Amossy, R. (2006). L'argumentation dans le discours. Paris : Armand Colin.

Bardin, L. (1993). L'analyse de contenu. Paris : Presses Universitaires de France.

Danblon, E. (2005). La fonction persuasive: Anthropologie du discours rhétorique. Origines et actualité. Paris : Armand Colin

Goldberg, M. (2005). Enjeux éthiques dans la formation épistémologique des étudiants en sciences de la vie - L'étude des théories de la bioénergétique. In UCIS (dir.), Actes du Colloque «Questions de pédagogie dans l'enseignement supérieur : Nouveaux contextes, nouvelles compétences » (p. 263-270). Villeneuve d'Ascq : UCIS.

Goldberg, M., Kraska, G. et Souchard, M. (2007). La discussion critique du contenu éthique et scientifique des manuels universitaires de biochimie. In Frenay, M., Wouters, P. et Raucent, B. (dir.), Actes du Colloque « Questions de pédagogies dans l'enseignement supérieur. Les pédagogies actives: enjeux et conditions» (p. 147-157). Louvain la Neuve : Presses Universitaires de Louvain.

Goldberg, M., Vinial, S. et Kraska, G. (2008). Les étudiants en sciences confrontés à l'analyse du discours. Communication présentée au Colloque « Questions de pédagogies dans l'enseignement supérieur. Enseigner, étudier dans le supérieur : pratiques pédagogiques et finalités éducatives ", 18 au 20 juin 2008, Brest.

Houdebine, L.-M. (2000) OGM. Le vrai et le faux. Paris : Le Pommier.

Kuntz, M. (2006). Les OGM, l'environnement et la santé. Paris : Ellipses.

Moirand, S. (2007). Les discours de la presse quotidienne. Paris : Presses Universitaires de France.

Oury, J.-P. (2006). La Querelle des OGM. Paris : Presses Universitaires de France.

Perelman, C. (1977). L'empire rhétorique. Rhétorique et argumentation. Paris : Vrin

Robin, M.M. (2008). Le monde selon Monsanto. Paris : La Découverte.

Seralini, G.E. (2004). Ces OGM qui changent le monde. Paris : Flammarion.

\section{NOTES}

1. «Cette situation, loin d'être imaginaire, s'est produite par exemple au Brésil, avant que le gouvernement de Lula décide de légaliser les importations pour régulariser le trafic qui avait lieu entre les paysans brésiliens et argentins ». 


\section{AUTEURS}

\section{MICHEL GOLDBERG}

Maître de conférences en biochimie à l'Université de La Rochelle, il travaille dans le laboratoire « Littoral, environnement et sociétés » et dirige un groupe de recherche pluridisciplinaire sur l'analyse du discours dans des documents à thème scientifique (le GREADIS). Il publie des recherches sur le développement de la pensée critique dans la formation universitaire et met en pratique des méthodes de pédagogie active dans le cadre des enseignements de biochimie et d'éthique.

\section{GRÂCE KRASKA}

Ingénieure d'études recherche et formation. Son activité est centrée sur les questions d'ingénierie de formation et d'ingénierie pédagogique ; plus précisément, les questions de professionnalisation des technologies de l'information et de la communication pour l'enseignement (TICE), l'e-pédagogie et l'accompagnement des enseignants à l'utilisation de la plateforme pédagogique Moodle. Elle a collaboré à des projets impliquant des établissements du secondaire. Elle travaille à @cTICE, la cellule d'Accompagnement aux TICE de l'Université de La Rochelle. 